\title{
Análisis del costo-utilidad de los nuevos fármacos para tratamiento del cáncer de próstata metastásico resistente a la castración
}

Velasco-Roces $L^{1}$, Rubio $T^{2}$

\section{Resumen}

OBJETIVO: estudiar el costo-utilidad en España de los diferentes fármacos para tratamiento del cáncer de próstata metastásico resistente a la castración (abiraterona, cabazitaxel, mitoxantrona y docetaxel) en pacientes que evolucionaron a un régimen de quimioterapia con docetaxel.

MATERIALES Y MÉTODOS: estudio descriptivo de corte longitudinal efectuado en pacientes con cáncer de próstata metastásico resistente a la castración. El costo-eficacia incremental para los diferentes fármacos se expresa en euros por años de vida ajustados por calidad para cáncer de próstata metastásico resistente a la castración. Para la estimación de los años de vida ajustados por calidad sólo se tuvieron en cuenta los costos directos y las utilidades se extrajeron de la bibliografía y se obtuvieron mediante estimaciones indirectas. Para la perspectiva del pagador se estimó un horizonte temporal de 18 meses y el índice de precios al consumidor en España, correspondiente a 2014.

RESULTADOS: cabazitaxel y abiraterona son los tratamientos más efectivos y más costosos. El costo-utilidad incremental de docetaxel, abiraterona, enzalutamida y cabazitaxel, tomando como referencia mitoxantrona, fue de $-3.581,41.720,98.845$ y 118,788 euros por años de vida ajustados por calidad, respectivamente. Mitoxantrona es dominado por docetaxel, fármaco tomado como referencia en este estudio.

CONCLUSIONES: con base en nuestro modelo, abiraterona es un tratamiento costo-efectivo para pacientes con cáncer de próstata metastásico resistente a la castración, enzalutamida y cabazitaxel resultaron opciones no costo-efectivas.

PALABRAS CLAVE: cáncer de próstata metastásico, cáncer de próstata metastásico resistente, docetaxel.
${ }^{1}$ Servicio de Farmacia, Hospital Universitario Central de Asturias, Oviedo, España.

${ }^{2}$ Servicio de Farmacia, Hospital de Jarrio, Navia. Servicio de Salud del Principado de Asturias, Oviedo, España.

Recibido: agosto 2016

Aceptado: enero 2017

Correspondencia Lucía Velasco Roces lucia.velasco@sespa.es

Este artículo debe citarse como

Velasco-Roces L, Rubio T. Análisis del costo-utilidad de los nuevos fármacos para tratamiento del cáncer de próstata metastásico resistente a la castración. Rev Mex Urol. 2017 ene;77(1):26-35. 


\section{Cost-utility analysis of new drugs for the treatment of metastatic castration- resistant prostate cancer}

Velasco-Roces $\mathrm{L}^{1}$, Rubio $\mathrm{T}^{2}$

\begin{abstract}
BACKGROUND: Prostate cancer is the most frequent tumor in men, followed by lung cancer. Pharmacologic treatment in patients with metastatic prostate cancer is palliative and its goal is to increase survival and quality of life. Between 10 and $20 \%$ of the patients will develop castration resistance within the first five years. It is recommended in all cases to maintain hormonal blockade associated with a new hormonal maneuver (androgen administration or withdrawal, extratesticular androgen synthesis, etc.) or others, such as chemotherapy.
\end{abstract}

OBJECTIVE: To carry out a cost-utility analysis in Spain of the different existing alternatives for the treatment of metastatic castration-resistant prostate cancer in patients with disease progression after a regimen of chemotherapy based on docetaxel.

MATERIALS AND METHODS: The incremental cost-effectiveness ratio of the different drugs was expressed as euros for qualityadjusted life years in metastatic castration-resistant prostate cancer. For the quality-adjusted life year estimate, only the direct costs were taken into account and the health utilities were retrieved from the literature and obtained through indirect estimates. The perspective of the payer was considered, along with a temporary horizon of 18 months, and the inflation rate in Spain corresponding to costs published up to 2014 .

RESULTS: Cabazitaxel and abiraterone were the most effective treatments, as well as the most expensive. Using mitoxantrone as the reference, the incremental cost-utility of docetaxel, abiraterone, enzalutamide, and cabazitaxel was -3,581, 41,720, 98,845, and 118,788 euros per quality-adjusted life year, respectively. Docetaxel was dominant in relation to mitoxantrone, the drug used as the reference in our study.

CONCLUSIONS: Based on our model, abiraterone is a cost-effective treatment in patients with metastatic castration-resistant prostate cancer, whereas enzalutamide and cabazitaxel are not.

KEY WORDS: Metastatic prostate cancer; Resistance; Docetaxel
${ }^{1}$ Servicio de Farmacia, Hospital Universitario Central de Asturias, Oviedo, España. ${ }^{2}$ Servicio de Farmacia, Hospital de Jarrio, Navia.

Servicio de Salud del Principado de Asturias, Oviedo, España.

Correspondence Lucía Velasco Roces lucia.velasco@sespa.es 


\section{ANTECEDENTES}

El cáncer de próstata es el primer tumor en frecuencia entre los hombres, seguido por el cáncer de pulmón. En España se diagnostican más de 25,000 casos anuales.

En la mayoría de los pacientes el diagnóstico se establece en estado asintomático y con el tumor localizado, sólo entre $10-20 \%$ se diagnostican en formas metastásicas.

El tratamiento farmacológico de los pacientes con cáncer de próstata metastásico es paliativo para aumentar la supervivencia y la calidad de vida; la carencia androgénica es el tratamiento de elección. Durante los primeros cinco años entre $10-20 \%$ de los pacientes se tornan resistentes a la castración. ${ }^{1}$ El Prostate Cancer Clinical Trials Working Group 2 (PCWG2) define al cáncer de próstata metastásico resistente a la castración como: progresión tumoral después de una maniobra de castración eficaz, con concentraciones de testosterona plasmática menores de $50 \mathrm{ng} / \mathrm{dL}$ (1.7 nmol/L). El grupo de pacientes con cáncer de próstata metastásico resistente a la castración es el de varones completamente asintomáticos, con elevación del antígeno prostático específico como única manifestación de progresión, hasta los que tienen daño multiorgánico y gran deterioro del estado general. A lo anterior deben añadirse las comorbilidades y situaciones inherentes a una población de pacientes casi siempre ancianos, que en ocasiones contrasta con otros jóvenes y con excelente estado general. La recomendación común es mantener el bloqueo hormonal ${ }^{2}$ junto con una nueva maniobra hormonal (administración o retiro de antiandrógenos, inhibición de la síntesis extratesticular de andrógenos...) u otras, ${ }^{3}$ como la quimioterapia. Docetaxel ha demostrado ser eficaz en el aumento de la supervivencia global y de la calidad de vida, así como en la reducción del dolor y del antígeno prostático específico. En los últimos años se han autorizado diversos fármacos para el tratamiento del cáncer de próstata metastásico resistente a la castración: acetato de abiraterona, cabazitaxel y enzalutamida, entre otros, por lo que es muy importante una evaluación exhaustiva y un correcto posicionamiento de los mismos, debido a las importantes implicaciones clínicas y económicas.

El acetato de abiraterona ${ }^{4}$ es un inhibidor selectivo de la enzima $17 \alpha$-hidroxilasa/17,20-liasa (CYP17), indispensable para la biosíntesis de andrógenos en los tejidos testiculares, suprarrenales y prostáticos tumorales. Ha demostrado superioridad frente a placebo al aumentar la supervivencia global de los pacientes con cáncer de próstata metastásico resistente a la castración que han evolucionado a un régimen de quimioterapia con docetaxel global a 4.6 meses de los pacientes.

Enzalutamida $^{5}$ es un inhibidor de la señalización de los receptores androgénicos, inhibe la unión de la testosterona a estos receptores, la translocación nuclear de los mismos y la unión y activación del ADN por parte de los mismos a los receptores androgénicos. Tiene afinidad por el receptor androgénico 5-8 veces mayor que bicalutamida. En el ensayo Pivotal AFFIRM demostró una mediana de supervivencia global, en el grupo de enzalutamida, de 18.4 meses vs 13.6 meses en el grupo placebo de pacientes con cáncer de próstata metastásico resistente a la castración que habían evolucionado tras un régimen de quimioterapia con docetaxel. ${ }^{6}$

Cabazitaxel $^{7}$ es un derivado de los taxanos, y mitoxantrona ${ }^{8}$ es un derivado de la antracenodiona, que se une al ADN nuclear. En el ensayo Pivotal TROPIC ${ }^{9}$ cabazitaxel reportó una supervivencia global de 15.1 vs 12.7 meses con mitoxantrona. El retratamiento con docetaxel, en pauta intermitente, ${ }^{10}$ podría tener indicación en pacientes en quienes inicialmente es efectivo; es decir, sin resistencia. Eymard y su grupo ${ }^{11}$ analizaron la respuesta al retratamiento con docetaxel en 100 pacientes que inicialmente tuvieron una reacción aceptable. El 48\% de los pacientes tuvo una reducción del antígeno 
prostático específico mayor de 50\%, la mediana de supervivencia global fue de 16 (13-20) meses y la tasa de supervivencia a los dos años de $28.9 \%$. El retratamiento fue bien tolerado (6\% toxicidad hematológica grado III-IV). La mediana de duración del retratamiento fue de 3.7 (0.5-11.5) meses. En 2006 Ohlmann ${ }^{12}$ y sus coautores obtuvieron resultados similares en una cohorte de 25 pacientes.

El objetivo de este trabajo es estudiar el costo-utilidad en España de los diferentes fármacos para tratamiento del cáncer de próstata metastásico resistente a la castración (abiraterona, cabazitaxel, mitoxantrona y docetaxel) en pacientes que evolucionaron a un régimen de quimioterapia con docetaxel.

\section{MATERIALES Y MÉTODOS}

Estudio descriptivo de corte longitudinal efectuado en pacientes con cáncer de próstata metastásico resistente a la castración. Criterios de inclusión: pacientes con adenocarcinoma de próstata confirmado histológica o citológicamente; con enfermedad metastásica confirmada por gammagrafía ósea o lesiones metastásicas que no fueron hepáticas o viscerales; con evolución, durante o tras un régimen de quimioterapia con docetaxel, según los criterios del PCWG2 (dos incrementos consecutivos del valor de antígeno prostático específico separados al menos una semana y con un valor mayor de $2.0 \mathrm{ng} / \mathrm{mL}$ ) o evidencia radiográfica de progresión de acuerdo con los criterios de RECIST modificado; pacientes asintomáticos o levemente sintomáticos con puntuación entre 0-1 en BPI-SF; castrados quirúrgicamente o médicamente, con concentraciones de testosterona menores de 50 $\mathrm{ng} / \mathrm{dL}$; $\mathrm{ECOG} \leq 2$; potasio sérico normal, función renal, hepática hematológica adecuadas y parámetros bioquímicos predefinidos (albúmina $\geq 3 \mathrm{~g} / \mathrm{dL}$ ).

Para la evaluación económica se calculan los años de vida ajustados por calidad (AVAC) para los diferentes fármacos, considerando la posología y pauta señalada en la ficha técnica de: acetato de abiraterona (1.000 mg/24 h), enzalutamida ( $160 \mathrm{mg} / 24 \mathrm{~h}$ ), cabazitaxel $\left(25 \mathrm{mg} / \mathrm{m}^{2}\right.$ cada tres semanas), docetaxel $\left(75 \mathrm{mg} / \mathrm{m}^{2}\right.$ cada 21 días). En el caso del acetato de abiraterona, ${ }^{13}$ enzalutamida, ${ }^{14}$ cabazitaxe $l^{9}$ y mitoxantrona la efectividad se obtiene de los ensayos pivotales y en el caso del retratamiento con docetaxel de los estudios publicados. ${ }^{15}$ En los ensayos clínicos se incluyen pacientes con características comparables.

El cálculo de los años de vida ajustados por calidad solo incluye costos directos, las utilidades se obtienen de la bibliografía, mediante estimaciones indirectas.

El costo-utilidad incremental (CUI) se calcula con la fórmula:

$$
\frac{\text { CUI = Costo A-Costo B }}{\mathrm{AVAC}_{\mathrm{A}}-\mathrm{AVAC}_{\mathrm{B}}}
$$

$\mathrm{AVAC}_{\mathrm{A}}=$ años de vida ajustados por calidad conseguidos con $\mathrm{A}$.

$\mathrm{AVAC}_{\mathrm{B}}=$ años de vida ajustados por calidad conseguidos con $\mathrm{B}$.

\section{Eventos adversos}

El perfil de efectos adversos de las diferentes opciones terapéuticas para el tratamiento de cáncer de próstata metastásico resistente a la castración es diferente, en el Cuadro 2 se reúnen los de grado 3 y 4.

\section{Utilidades}

Las medidas de utilidad de los pacientes, para diferentes estados de salud, se obtuvieron de 
la bibliografía y las medidas, diferentes cuestionarios con una escala del 0 al 1 donde el 0 representa la muerte y el 1 el estado de perfecta salud. En el análisis en cada uno de los brazos de tratamiento se utilizan las utilidades aplicando los valores más bajos representativos del modelo (Cuadro 3).

\section{Recursos y costos}

El estudio se efectúa desde la perspectiva del pagador, incluyendo sólo los recursos y costos directos. Para calcular las dosis de quimioterapia intravenosa se utiliza una superficie corporal de $1.65 \mathrm{~m}^{2}$. En el caso de los fármacos de administración intravenosa se consideran también los costos de premedicación, según información reunida en la ficha técnica de los medicamentos, preparación y administración en el hospital de día. El costo total por ciclo es la suma de la premedicación, preparación y administración.

Los costos se obtienen de la bibliografía, expresados en dólares americanos (año 2012); para su conversión a euros se consideró el cambio dólar-euro en 2012 y el índice de precios al consumidor correspondiente a ese periodo en España.

Considerando un análisis determinista, los costos totales, la expectativa de vida (AVG) se calcularon los años de vida ajustados por calidad para los diferentes tratamientos y se analizaron en un horizonte temporal de 18 meses. Se optó por este horizonte temporal para estandarizar los diferentes ensayos pivotales, lo que no precisa aplicar tasas de descuento, según recomiendan las últimas guías publicadas. ${ }^{16-20}$

\section{RESULTADOS}

La supervivencia global es de 14.8, 18.4, 16 y 15.2 meses para abiraterona, enzalutamida retratamiento con docetaxel y cabazitaxel, respectivamente (Cuadro 1).

Entre los costos directos vinculados con eventos adversos sólo se tienen en cuenta los más importantes (Cuadro 4). Debido a la neutropenia que origina cabazitaxel se recomienda la profilaxis ${ }^{21}$ con factores estimulantes de colonias. La neutropenia originada incluye la hospitalización para $19 \%$ de los pacientes con neutropenia grado IIIIV y dos semanas de tratamiento profiláctico con factor estimulante para $100 \%$ de los pacientes que reciben una mediana de seis ciclos de tratamiento. ${ }^{22}$ Los pacientes tratados con mitoxantrona casi siempre son mínimamente tratados de la neutropenia grado III-IIV y no son tratados profilácticamente. Por lo tanto, se aplica el costo de $50 \%$ de los pacientes tratados con mitoxantrona.

Para acetato de abiraterona, el tratamiento de los eventos cardiacos grado III y IV incluyen electrocardiograma y hospitalización por arritmias y fibrilación ventricular (20\%).

En adición a los costos por efectos adversos se toma el costo de 15 cursos de radioterapia para paliar el dolor a los pacientes con dolor basal en el grupo de mitoxantrona (22.5\% de los pacientes en cada uno de los grupos). Se asume, basándose en los datos de los ensayos clínicos que cabazitaxel, mitoxantrona, enzalutamida y docetaxel ejercen un efecto paliativo, el cuál requiere menos tratamiento del dolor que prednisona sola. Los costos de la radiación se han estimado a partir de los datos publicados de la utilización de tratamientos del código CPT. ${ }^{23}$

Los datos de calidad de vida relacionada con la salud se incorporaron como valores de utilidad. La utilidad de los diferentes brazos de tratamiento se vio afectada por el perfil de eventos adversos, la tasa de respuesta y de progresión. Se asume que no hay diferencias en la utilidad inherente de los diferentes 
Cuadro 1. Fármacos para el tratamiento de cáncer de próstata metastásico resistente a la castración

\begin{tabular}{|c|c|c|c|c|c|}
\hline & Abiraterona & Enzalutamida & (1)Docetaxel & Mitoxantrona & Cabazitaxel \\
\hline Pauta & $\begin{array}{c}1000 \mathrm{mg} / 24 \mathrm{~h}+ \\
\text { prednisona } 10 \mathrm{mg} / 24 \mathrm{~h}\end{array}$ & $160 \mathrm{mg} / 24 \mathrm{~h}$ & $\begin{array}{c}75 \mathrm{mg} / \mathrm{m}^{2} \\
\mathrm{c} / 3 \text { semanas + } \\
\text { prednisona } 5\end{array}$ & $\begin{array}{c}12 \mathrm{mg} / \mathrm{m}^{2} \\
\mathrm{c} / 3 \text { semanas }\end{array}$ & $\begin{array}{c}25 \mathrm{mg} / \mathrm{m}^{2} \\
\mathrm{c} / 3 \text { semanas }+ \text { prednisona } \\
10 \mathrm{mg} / 24 \mathrm{~h}\end{array}$ \\
\hline Vía & Oral & Oral & Intravenosa & Intravenosa & Intravenosa \\
\hline $\begin{array}{l}\text { Supervivencia } \\
\text { global (meses) }\end{array}$ & 14.8 & 18.4 & 16 & 12.7 & 15.1 \\
\hline
\end{tabular}

(1) En aquellos pacientes que previamente hayan respondido a docetaxel.

Cuadro 2. Efectos adversos grado 3 ó 4 producidos por los diferentes fármacos según los estudios pivotales de los fármacos

\begin{tabular}{|c|c|c|c|c|c|}
\hline & $\begin{array}{l}\mathrm{ABI} \\
(\%)\end{array}$ & $\begin{array}{c}\text { ENZA } \\
(\%)\end{array}$ & $\begin{array}{c}\text { CABA } \\
(\%)\end{array}$ & $\begin{array}{c}\text { MITOX } \\
(\%)\end{array}$ & $\begin{array}{c}\text { DOCE } \\
(\%)\end{array}$ \\
\hline $\begin{array}{l}\text { Retención de } \\
\text { líquido y edema }\end{array}$ & 3 & & & & \\
\hline Hipocalemia & 4 & & & & \\
\hline Hipertensión & 1 & & & & \\
\hline $\begin{array}{l}\text { Alteraciones } \\
\text { cardiacas }\end{array}$ & 5 & 1 & & & \\
\hline $\begin{array}{l}\text { Infarto de } \\
\text { miocardio, angina }\end{array}$ & 1 & 1 & & & \\
\hline$\uparrow A L T$ & 4 & 2 & & & \\
\hline$\uparrow \mathrm{AST}$ & 3 & 1 & & & \\
\hline Neutropenia & & & 82 & 58 & 54 \\
\hline Leucopenia & & & 68 & 42 & \\
\hline Anemia & 7 & & 11 & 5 & 11 \\
\hline Fatiga & 9 & 6 & 5 & 3 & \\
\hline Crisis & & 1 & & & \\
\hline Diarrea & 1 & & 6 & 1 & \\
\hline
\end{tabular}

ABI: Acetato de Abiraterona, ENZA: Enzalutamida, CABA: Cabazitaxel, MITOX: Mitoxantrona, DOCE: Docetaxel

AST: Aspartato amino transferasa; ALT: Alanino amino transferasa

tratamientos. En cada uno de los brazos de tratamiento se aplicó la pérdida de utilidad por efectos adversos considerando el valor más bajo de utilidad asociado con los pacientes encontrado en la bibliografía. La probabilidad de estos eventos adversos se obtuvo de los ensayos clínicos.
Los años de vida ajustados por calidad se calcularon multiplicando las correspondientes utilidades por las expectativas de vida. Las tasas de costo-utilidad-incremental de las alternativas comparadas se obtuvieron teniendo como referencia la de menor utilidad, que es mitoxantrona (Cuadro 5).

La representación gráfica de los costos (eje Y) y de la utilidad (eje X) de las distintas opciones permite visualizar el costo-utilidad medio (CUIM). Tres de las cuatro alternativas: acetato de abiraterona, cabazitaxel y enzalutamida se encuentran en el primer cuadrante del plano costo-utilidad (Figura 1), mayor utilidad y mayor costo, por lo que la elección depende del valor costo-utilidad incremental que se esté dispuesto a pagar. El retratamiento con docetaxel se encuentra en el cuadrante II (mayor utilidad y menor costo).

\section{DISCUSIÓN}

Desde el punto de vista de los costos, docetaxel es el tratamiento de costo más bajo $(57,252$ euros), seguido de mitoxantrona (58,219 euros), abiraterona (70,735 euros) y cabazitaxel (109,298 euros) (Cuadro 4).

Los dos criterios existentes para tomar una decisión terapéutica luego de analizar el costo-utilidad son: el criterio del presupuesto y el criterio del precio máximo que se está dispuesto a pagar por una 
Cuadro 3. Medidas de utilidad de los pacientes para diferentes estados de salud

\begin{tabular}{|l|c|c|c|}
\hline Variable de utilidad & Utilidad & Medida de utilidad & Referencia \\
\hline Dolor óseo & 0.43 & HUI & Konski, et al $(2009)^{17}$ \\
\hline Alteraciones cardiacas & 0.51 & EQ-5D & Dyer M, et al $(2010)^{18}$ \\
\hline Dolor & 0.55 & QWB & Krahn M, et al $(2003)^{19}$ \\
\hline Neutropenia & 0.57 & SG & Nafees, et al $(2008)^{20}$ \\
\hline Radiación & 0.62 & QWB & Krahn M, et al $(2003)^{19}$ \\
\hline Enfermedad metastásica & 0.62 & QWB & Krahn M, et al $(2003)^{19}$ \\
\hline Radiación & 0.67 & QWB & Krahn M, et al $(2003)^{19}$ \\
\hline No Dolor & 0.69 & QWB & Krahn M, et al $(2003)^{19}$
\end{tabular}

Cuadro 4. Costos directos asociados al tratamiento con cada una de las alternativas del tratamiento evaluado ${ }^{24}$

\begin{tabular}{|c|c|c|c|c|c|}
\hline & $\begin{array}{c}\text { Acetato de } \\
\text { Abiraterona }\end{array}$ & Enzalutamida & Cabazitaxel & Mitoxantrona & Docetaxel \\
\hline Costo fármaco $(€)$ & $33.289(+)$ & $36.194(+)$ & $34.427(+)$ & $1.962(+)$ & $995(+)$ \\
\hline Neutropenia grado III/IV $(€)$ & & & $36.484(* *)$ & $12.161\left(^{*}\right)$ & $12.161\left(^{*}\right)$ \\
\hline Neutropenia grado 0-II (€) & & & $21.079(* *)$ & & \\
\hline Alteraciones cardiacas grado III/IV $(€)$ & $8.408(\#)$ & & & & \\
\hline Radiación por elevado dolor basal (€) & $1.257(\&)$ & $1.257(\&)$ & $1.257(\&)$ & $1.257(\&)$ & $1.257(\&)$ \\
\hline Crisis epilépticas (€) & & 2.370 (¥) & & & \\
\hline Muerte asociada a la hospitalización (€) & $66.887(¥ ¥)$ & $66.887(¥ ¥)$ & $66.887(¥ ¥)$ & $66.887(¥ ¥)$ & $66.887(¥ ¥)$ \\
\hline 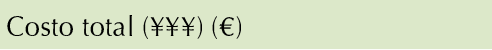 & 70.735 & 90.838 & 109.298 & 58.219 & 57.252 \\
\hline
\end{tabular}

(+) Incluye costo fármaco (PVL+4\% IVA)+ premedicación+administración en hospital de día. Se ha tenido en cuenta la mediana de ciclos obtenida de estudios pivotales, para 10,1 mediana de tratamiento con acetato de abiraterona, 6 ciclos cabazitaxel y 4 de mitoxantrona datos del estudio, 8,3 meses enzalutamida y 4 ciclos de retratamiento con docetaxel

(*) Dos semanas de factor estimulante de colonias por ciclo, para una media de cuatro ciclos, una hospitalización para el 9,5\% de los pacientes y el seguimiento médico después del alta.

$\left.{ }^{(* *}\right)$ Dos semanas de factor estimulante de colonias por ciclo, para una media de seis ciclos, una hospitalización para el 19\% de los pacientes y una visita al doctor

$\left.{ }^{* * *}\right)$ Dos semanas de tratamiento con factor estimulante de colonias por ciclo para todos los pacientes (6 ciclos)

(\#) Costo medio del tratamiento de arritmias (89\%) y paradas cardiacas, hospitalización y 5 electrocardiogramas.

(\&) Un ciclo de radiación con 15 tratamientos para la mitad de los pacientes con dolor basal en el grupo de tratamiento.

(¥) Para una mediana de cuatro episodios de crisis limitantes y un episodio de status epiléptico que requiere hospitalización.

(¥¥) Costo medio de la hospitalización por efectos adversos (neutropenia y eventos cardiacos) para una estancia media de 22 días.

(¥¥¥) Son datos de 2012 se ha considerado el cambio Dólar americano Euro y el IPC de ese año, para ajustar a 2014.

Se han utilizado las probabilidades extraídas de los diferentes estudios.

utilidad adicional. En España no existe un valor oficial, pero se cita generalmente como referencia el umbral de 30,000 euros-años de vida ajustados por calidad, aunque en algunas publicaciones se sugieren umbrales superiores que están en torno a los 45,000 euros-años de vida ajustados por calidad. En el caso de los medicamentos contra el cáncer se duda de que los años de vida ajustados por calidad reflejen de manera adecuada el valor social de estos medicamentos, debido a las características específicas que concurren en los tratamientos oncológicos en fases avanzadas de la enfermedad (el potencial de mejora de la salud suele ser escaso, las ganancias en salud suelen 
Cuadro 5. Coste Utilidad Incremental. Variables continuas. Mediana de supervivencia

\begin{tabular}{|l|c|c|c|c|}
\hline Fármaco & $\begin{array}{c}\text { Supervivencia } \\
\text { global (años) }\end{array}$ & AVACs & $\begin{array}{c}\text { Costo incremental } \\
(€)\end{array}$ & $\begin{array}{c}\text { Costo } \\
\text { Utilidad Incremental (CUI) } \\
€ / \text { AVAC }\end{array}$ \\
\hline $\begin{array}{l}\text { Mitoxantrona } \\
\text { Retratamiento } \\
\text { Con docetaxel }\end{array}$ & 1.05 & 0.43 & ------------ & -3.581 \\
\hline $\begin{array}{l}\text { Acetato } \\
\text { de abiraterona }\end{array}$ & 1.33 & 0.70 & -967 & 41.720 \\
\hline Enzalutamida & 1.23 & 0.73 & 12.516 & 98.845 \\
\hline Cabazitaxel & 1.25 & 0.76 & 32.619 & 118.788 \\
\hline
\end{tabular}

Se ha tomado como referencia el fármaco con menor número de QALYs que es mitoxantrona.

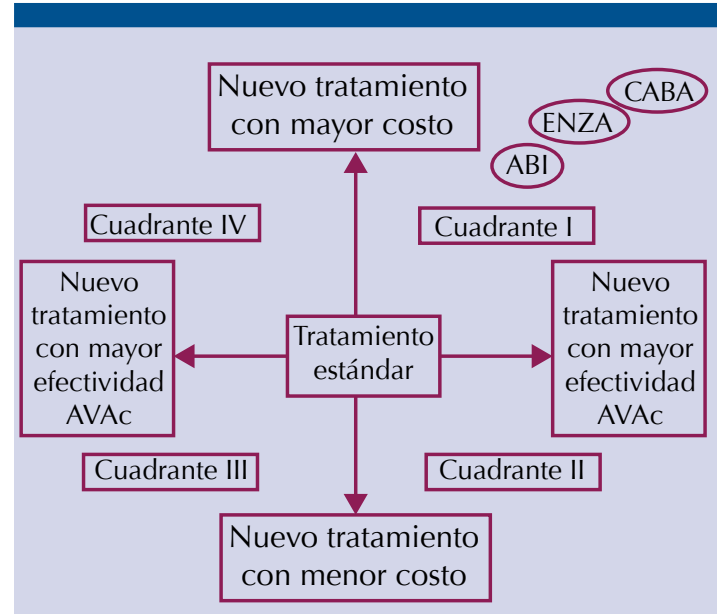

ABI: Acetato de Abiraterona, ENZA: Enzalutamida, DOCE: Docetaxel. Se ha considerado como estándar el tratamiento con mitoxantrona.

Figura 1. Representación del plano costo-utilidad para las diferentes alternativas analizadas.

tener lugar sólo durante un periodo corto y los pacientes a quienes se dirigen los tratamientos se encuentran generalmente en la etapa final de sus vidas.) Para dar respuesta a esta situación, en enero de 2009, el National Institute for Health and Clinical Excellence (NICE) puso en práctica una serie de criterios para considerar los tratamientos "al final de la vida" (end-of-life treatments). El costo-utilidad incremental que el NICE acepta ahora para este tipo de situaciones es superior al resto de tecnologías, 48,000-60,000 euros por años de vida ajustados por calidad ganados. Los criterios de tratamiento para el final de la vida pueden aplicarse siempre que: la esperanza de vida de los pacientes a tratar sea inferior a 24 meses, que el medicamento ofrezca un aumento en la supervivencia superior a tres meses, que el tratamiento esté indicado para un grupo de pacientes relativamente reducido (normalmente no excedan los 7000 pacientes nuevos por año en la población británica) y que no exista alternativa de similar efectividad en el sistema sanitario.

El costo-utilidad incremental, respecto a mitoxantrona, es de $41,720,98,845$ y 118,788 euros-años de vida ajustados por calidad para abiraterona, enzalutamida y cabazitaxel, respectivamente. Docetaxel, con un costo-utilidad incremental -3,581 euros-años de vida ajustados por calidad tiene un comportamiento dominante al tener una mayor utilidad y menor costo respecto a mitoxantrona; no obstante esta opción de tratamiento solo sería adecuada para los pacientes sin resistencia inicial a docetaxel y en los que el tiempo de evolución ha sido entre 3 y 6 meses.

Teniendo en cuenta que el costo-utilidad incremental es de 98,956 euros-años de vida ajustados por calidad para enzalutamida $y$ 
118,788 euros-años de vida ajustados por calidad para cabazitaxel, ambos superan ampliamente el precio máximo que se está dispuesto a pagar para los tratamientos "al final de la vida" (48,000-60,000 euros). Cabazitaxel debido a los elevados costos del tratamiento de sus efectos adversos, en particular la neutropenia que origina, hace que el costo-utilidad incremental sea muy elevado $(118,788$ euros-años de vida ajustados por calidad), lo mismo sucede con enzalutamida en el que el costo-años de vida ajustados por calidad está próximo a los 100,000 euros.

No obstante, estos resultados deberían tomarse como una aproximación o estimación puesto que debería completarse con un análisis de sensibilidad probabilístico, que escapa al planteamiento de este trabajo.

Otro aspecto importante a tener en cuenta es que en los estudios pivotales con abiraterona y cabazitaxel fue necesaria la prednisona; en el caso de enzalutamida es opcional porque es un inhibidor del receptor androgénico y no exige la administración concomitante de este fármaco. Los estudios de retratamiento con docetaxel son prospectivos, con un número reducido de pacientes por lo que la evidencia es limitada.

Para la evaluación económica sólo se han tenido en cuenta los efectos adversos más importantes y los costos directos.

En pacientes con cáncer de próstata metastásico resistente a la castración que hayan recibido un tratamiento previo con docetaxel tanto acetato de abiraterona, cabazitaxel, enzalutamida como el retratamiento con docetaxel tienen unos resultados de supervivencia global similares; no obstante, los valores de utilidad y los costos asociados a los tratamientos de los eventos adversos hace que tengan valores muy dispares en costo-utilidad incremental, teniendo como referencia mitoxantrona, que oscilan entre $-3,581$ euros para docetaxel y 118,788 euros para cabazitaxel.

El acetato de abiraterona tiene un costo-utilidad incremental, respecto a mitoxantrona, que está dentro de los criterios para el final de la vida, de precio máximo que se está dispuesto a pagar por unidad de utilidad adicional. Con base en los resultados de esta investigación, ni cabazitaxel ni enzalutamida parecen ser tratamientos costo-útiles en la población de estudio. Un reciente análisis de costo-efectividad publicado por el NICE concluye que cabazitaxel no es costo-efectivo y no es recomendado. ${ }^{24}$ Aunque la supervivencia global obtenida en los ensayos clínicos es ligeramente superior con cabazitaxel que con acetato de abiraterona, debido al perfil de seguridad, en particular la neutropenia que origina hace que la relación costo-utilidad sea muy desfavorable para el fármaco.

Con base en los resultados de este estudio los pacientes a quienes se haya administrado docetaxel sin demostrar resistencia inicial, con un tiempo hasta la progresión entre 3 y 6 meses el retratamiento con docetaxel es la opción que debiera considerarse de elección.

\section{REFERENCIAS}

1. Kirby M, Hirst C, Crawford ED. Characterizing the castrationresistant prostate cancer population: a systematic review. Int J Clin Pract 2011; 65: 1180-92.

2. Taylor CD, Elson P, Trump DL. Importance of Continued Testicular Suppression in Hormone-Refractory Prostate Cancer. J Clin Oncol 1993; 11:2167-21812.

3. Arranz JA, González R, López C, Sabin P, Soria A, Jerez Y. Tratamiento del paciente con carcinoma de próstata en progresión bioquímica resistente a castración Arch. Esp. Urol. 2012; 65 (1): 185-192

4. Ficha técnica Zytiga ${ }^{\circledR}$. Disponible en:

5. http://www.ema.europa.eu/docs/es_ES/document_library/EPAR__Product_Information/human/002321/ WC500112858.pdf. [Acceso Mayo 2014].

6. Ficha técnica $X$ tandi $^{\circledR}$. Disponible en:

7. http://www.ema.europa.eu/docs/es_ES/document_library/EPAR__Product_Information/human/002639/ WC500144996.pdf. [Acceso Mayo 2014]. 
8. Scher HI, Fizazi K, Saad F, Taplin ME, Sternberg CN, Miller $\mathrm{K}$, et al. Increased survival with enzalutamide in prostate cancer after chemotherapy. N Engl J Med. 2012 Sep 27; 367(13):1187-97.

9. Ficha técnica Jevtana ${ }^{\circledR}$. Disponible en:

10. http://www.ema.europa.eu/docs/es_ES/document_library/EPAR_-_Product_Information/human/002018/ WC500104764.pdf. [Acceso Mayo 2014].

11. Ficha técnica Novantrone ${ }^{\circledR}$. Disponible en

12. http://www.aemps.gob.es/cima/pdfs/es/ft/66166/ FT_66166.pdf [Acceso Mayo 2014].

13. de Bono JS, Oudard S, Ozguroglu M, Hansen S, Machiels JP, Kocak, et al. Prednisone plus cabazitaxel or mitoxantrone for metastatic castration-resistant prostate cancer progressing after docetaxel treatment: a randomised open-label trial. Lancet. 2010 Oct 2; 376(9747):1147-54.

14. Beer TM, Garzotto M, Henner WD, Eilers KM, Wersinger EM. Multiple cycles of intermittent chemotherapy in metastatic androgen-independent prostate cancer. Br J Cancer 2004;91(8):1425-27.

15. Eymard JC1, Oudard S, Gravis G, Ferrero JM, Theodore $C$, Joly $F$, et al. Docetaxel reintroduction in patients with metastatic castration-resistant docetaxel-sensitive prostate cancer: a retrospective multicentre study. BJU Int. 2010 Oct; 106(7):974-8.

16. Ohlmann C, Ozgur E, Wille S, Engelman U, Heidenreich A. Second-line chemotherapy with docetaxel for prostatespecific antigen relapse in men with hormone refractory prostate cancer previously treated with docetaxel based chemotherapy. Eur Urol Suppl 2006; 5(2):93.

17. de Bono JS, Logothetis CJ, Molina A, Fizazi K, North S, Chu $L$, et al. Abiraterone and increased survival in metastatic prostate cancer. N Engl J Med. 2011 May 26; 364(21):19952005.

18. Scher HI, Fizazi K, Saad F, Taplin ME, Sternberg CN, Miller $\mathrm{K}$, et al. Increased survival with enzalutamide in prostate cancer after chemotherapy. N Engl J Med. 2012 Sep 27; 367(13):1187-97.

19. López Bastida J, Oliva J, Antoñanzas F, García-Altés Ab, Gisbert R, Mar J, Puig-Junoy J, et al. Propuesta de Guía para la evaluación económica aplicada a las tecnologías sanitarias. Madrid: Plan Nacional para el SNS del MSC. Servicio de Evaluación del Servicio Canario de la Salud: Informes de Evaluación de tecnológicas Sanitarias SESCS
2008 № 2006/22. Disponible en http://aunets.isciii.es/ ficherosproductos/132/Memoria Fibal.pdf.

20. Konski A, James J, Hartsell W, Leibenhaut MH, MD, Janjan $\mathrm{N}$, Curran W, et al. Economic Analysis of radiation Therapy Oncology Group (RTOG). Multiple versus single fraction radiation treatment of patients with bone metastases. Am J Clin Oncol 2009; 32:6.

21. Dyer M, Goldsmith K, Sharples L and Buxton M. A review of health utilities using the EQ-5D in studies of cardiovascular disease. Health and quality of life Outcomes 2010; 8:13.

22. Krahn M, Ritvo P, Irvine J, Tomlinson G, Bremner KE, Bezjak $A$, et al. Patient and community preferences for outcomes in prostate cancer: Implications for clinical Policy. Medicale Care 2003; 41:12.

23. Nafees B1, Stafford M, Gavriel S, Bhalla S, Watkins J. Health state utilities for non small cell lung cancer. Health and Quality of Life Outcomes 2008;6:84.

24. Freifeld AG, Bow EJ, Sepkowitz KA, Boeckh MJ, Ito JI, Mullen CA. Clinical Practice Guideline for the use of antimicrobial agents in neutropenic patients with cancer: 2010 update by the Infectious Diseases Society of America. Clinical infectious Diseases 2011; 52: e56-e93.

25. Ozguroglu M, Oudard S, Sartor O, Hansen S, Machiels JH, Shen L, et al. Effect of G-CSF prophylaxis on the ocurrence of neutropenia in men receiving cabazitaxel plus prednisone for the treatment of metastatic castration-resistant prostate cancer ( $\mathrm{mCRPC}$ ) in the TROPIC study Genitourinary Symposium. J Clin Oncol 29: 2011 (Suppl 7; abstr 144)

26. Konski A, Watkins-Bruner D, Fergenberg $S$, et al. Using decision analysis to determine the cost-effectiveness of intensivity-modulated radiation therapy in the treatment of intermediate risk prostate cancer. Int J Radiat Onc Biol Phys; 2006 Oct 1;66(2):408-15.

27. Zhong L1, Pon V, Srinivas S, Nguyen N, Frear M, Kwon $S$, Gong $C$ et al.. Therapeutic options in docetaxel-refractory metastatic castration-resistant prostate cancer: a costeffectiveness analysis. PLoS One. 2013 May 22;8(5).

28. National Institute for Health and Clinical Excellence (2012) Cabazitaxel for hormone-refractory mestastatic prostate cancer previously treated with docetaxel-containing regimen: Final appraisal determination. Disponible http:// guidance.nice.org.uk/TA/Wave23/31/FAD/FinalAppraisalDetermination/ pdf/English. [Acceso Mayo 2014]. 